\title{
Violencia en el entorno laboral del trabajo sexual y consumo de sustancias en mujeres mexicanas
}

\author{
Eva Ma. Rodríguez, ${ }^{1}$ Patricia Fuentes, ${ }^{1}$ Luciana Ramos-Lira, ${ }^{1}$ Rafael Gutiérrez, ${ }^{1}$ Eunice Ruiz'
}

Artículo original

\section{SUMMARY}

\section{Objective}

The aim of this paper is to explore in women sex workers the violence they experience in the exercise of their activity, and if it is associated with alcohol use and the consumption of other substances such as cocaine, mariguana and tobacco.

\section{Method}

This is a descriptive, non-experimental study, developed with a nonrandom sample of 103 sex workers selected for convenience type field. The selection criteria were being between 18 and 65 years of age, being a sex worker, and literate. An expressly designed instrument consisting of a semi-structured interview was used.

\section{Results}

Regarding the workplace, $50.8 \%$ of them worked in bars, $39.0 \%$ on the streets, and $10.2 \%$ in cabarets. More than a half reported having suffered violent experiences in the workplace; of these, $95.8 \%$ experienced physical violence, $26.0 \%$, sexual violence, and $24.0 \%$, emotional violence. Regarding the actors involved in violent episodes, they were other sex workers $(97 \%)$, customers $(77.2 \%)$, owners or employees of bars $(30.2 \%)$, and police $(25.0 \%)$. Regarding substance use, $98 \%$ of the sex workers reported having consumed alcohol in the past month; $23.3 \%$, cocaine; and $11.7 \%$, marijuana. Likewise, $40 \%$ smoked tobacco. Seventy-five percent of the sex workers reported being a high drinker. Most of the women reported that customers generally pressed them in to drinking alcohol and a high percentage reported that they consumed it because of the demands of bar or cabaret managers.

The experiences of workplace violence were associated with cocaine use $\left(\chi^{2}=6.417[100,1] \mathrm{df} p<.05\right)$ and tobacco $\left(\chi^{2}=7.486\right.$ [100.1] $\mathrm{gl} \mathrm{p}<.01)$. This association remained if violence had occurred in the last month. Alcohol consumption was associated with experiences of physical violence $\left(\chi^{2}=5.180\right.$ [100.1] $\left.\mathrm{gl} p<.05\right)$, and emotional abuse $\left(\chi^{2}=4.514[100.1] \mathrm{gl} p<.05\right)$.

\section{Discussion}

The results show that violence is a common experience in the work environment of these women, and that this is carried out by multiple actors. Findings highlight that sex workers point out to other sex workers as those who exercise more violence. High alcohol use and the use of other drugs are associated with this violence, requiring future studies to explore in-depth this link. The findings are discussed, with recommendations for the care of these problems in female sex workers.

Key words: Work setting, violence, sex workers, addictions.

\section{RESUMEN}

\section{Objetivo}

Conocer la violencia que viven las trabajadoras sexuales en el ejercicio de su actividad y si ésta se asocia con su consumo de alcohol y otras sustancias.

\section{Método}

Es un estudio de campo tipo no experimental, descriptivo, con una muestra no probabilística de 103 trabajadoras sexuales seleccionadas por conveniencia. Se utilizó un instrumento diseñado ex profeso, consistente en una entrevista semi-estructurada.

\section{Resultados}

El $50.8 \%$ trabajaba en un bar, el $39.0 \%$ en la calle y el $10.2 \%$ en un cabaret. Más de la mitad $(52.4 \%)$ refirió haber vivido experiencias violentas en el lugar de trabajo; de éstas, el $95.8 \%$ sufrió violencia física, el $26.0 \%$ violencia sexual y el $24.0 \%$ violencia emocional. Respecto a los actores involucrados en episodios violentos, en el $97.9 \%$ de los casos fueron compañeras de trabajo, en el $77.2 \%$, clientes; en el $30.2 \%$, propietarios o empleados de los bares y en el $25.0 \%$, la policía.

El $98 \%$ de las trabajadoras sexuales reportaron haber consumido alcohol en el último mes, el $23.3 \%$ cocaína y el $11.7 \%$ mariguana. Cabe señalar que $40 \%$ fuma tabaco. El $75 \%$ de las trabajadoras sexuales se reporta como bebedora alta. El $97 \%$ informó que generalmente los clientes las presionan para beber alcohol y un porcentaje similar refirió que consumen por las exigencias de los encargados de los lugares de trabajo.

Las experiencias de violencia en el trabajo se asociaron con

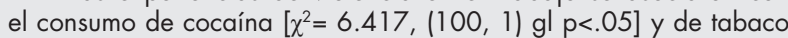
$[2=7.486(100,1) \mathrm{gl} \mathrm{p}<.01]$. Esta asociación se mantuvo si la violencia había ocurrido en el último mes. El consumo de alcohol se relacionó con las experiencias de violencia física $\left[\chi^{2}=5.180(100,1) \mathrm{gl}\right.$ $p<.05], y$ de violencia emocional $\left[\chi^{2}=4.514(100,1) \mathrm{gl} p<.05\right]$.

\section{Discusión}

Los resultados muestran que la violencia es una experiencia frecuente en su ambiente de trabajo, y es ejercida por múltiples actores; destaca el que se mencione a otras trabajadoras sexuales como quienes la ejercen, lo que no se reporta en la bibliografía. El consumo de alcohol y otras drogas se asocia con esta violencia, por lo que debe explorarse más este vínculo en futuros estudios. Se discuten los hallazgos, haciendo recomendaciones para la atención de esta problemática en las mujeres.

Palabras clave: Ambiente laboral, violencia, trabajadoras sexuales, consumo de alcohol, consumo de drogas.

Dirección de Investigaciones Epidemiológicas y Psicosociales. INPRFM.

Correspondencia: Dra. Luciana Ramos-Lira. Dirección de Investigaciones Epidemiológicas y Psicosociales. Instituto Nacional de Psiquiatría Ramón de la Fuente Muñiz. Calz. México-Xochimilco 101, San Lorenzo Huipulco, Tlalpan, 14370, México, D.F. Tel: 4160 -5186. E-mail: ramosl@imp.edu.mx 


\section{INTRODUCCIÓN}

El trabajo sexual incluye a "mujeres, varones y transexuales adultos y jóvenes que reciben dinero o bienes a cambio de sus servicios sexuales, ya sea de forma regular u ocasional, y que pueden definir o no conscientemente estas actividades como generadoras de ingresos". ${ }^{1}$ En algunos casos es una actividad temporal que suele realizarse cuando el sexo se intercambia por necesidades básicas o por protección (lo que se denomina sexo por sobrevivencia). Puede realizarse en ámbitos organizados formalmente como bares, table dance, clubes nocturnos, hoteles, burdeles, casas de citas o al prestar servicios a domicilio en eventos sociales o fungir como damas de compañía (escorts); o de manera flexible cuando se ejerce en la calle o como un autoempleo, ${ }^{2}$ teniendo cada uno de estos espacios sus propias reglas y condiciones laborales.

El trabajo sexual por sí mismo puede tener repercusiones importantes en la salud física y mental de quienes lo ejercen, dadas las múltiples situaciones que cotidianamente posicionan a las trabajadoras sexuales en condiciones de vulnerabilidad por diversos motivos. Los aspectos socioculturales relacionados con el estigma y la discriminación generan graves violaciones a sus derechos humanos y abusos que rara vez se consideran en la discusión sobre la violencia basada en el género. ${ }^{3}$ Así pues, mientras no haya un cambio de actitudes respecto a la percepción que se tiene de las mujeres que realizan trabajo sexual y persista el discurso que las construye como "desechables", es posible que persista su situación de minoría sexual vulnerable, al ser criminalizadas por su sola actividad y por ser vistas como ciudadanas de segunda. ${ }^{4}$

Alrededor del mundo las tasas de mortalidad estandarizadas para trabajadoras sexuales es seis veces más alta que en la población general (y es 18 veces más alta por asesinato); la más elevada para cualquier grupo de mujeres. ${ }^{5}$ Como señalan Goodyear y Cusick, ${ }^{5}$ "la muerte y la violencia no son más que parte de un espectro de la persistente morbilidad física y emocional".

En particular, son las trabajadoras sexuales que laboran en las calles las que se enfrentan a una gama de problemas sociales que se intersectan: la pobreza, el encarcelamiento, el abuso de sustancias, el riesgo de infección del virus de inmunodeficiencia humana, historias de abuso infantil y de violencia de pareja. . $^{5}$

Estas mujeres tienen un riesgo mayor de sufrir asaltos, violación y otras formas de violencia física. ${ }^{3,8-10}$ Asimismo, sus actividades están enclavadas en los mismos espacios donde ocurre la violencia callejera y otras modalidades delictivas, ${ }^{8}$ lo que ha llevado a pensar en que viven un ciclo de violencia difícil de parar, que se inicia desde la infancia. ${ }^{4}$

Esta violencia ha sido atribuida también a la inequidad de género y la discriminación contra las mujeres, así como a los intentos de muchos hombres para ejercer control sexual sobre ellas. El trato de mercancía sexual implica el uso de la violencia sexual, entendida ésta como la imposición de una relación sexual indeseable; de posturas, gestos, actos o formas de vestir; el abuso y la violación, los insultos y las acusaciones durante las relaciones sexuales así como la venganza, el chantaje, las amenazas y palizas si hay resistencia a las relaciones sexuales. A esto se agregan otras discriminaciones que incrementan la violencia, como las de clase o raza. ${ }^{8}$

Por ello, no es de extrañar, que a nivel mundial, entre el 50 al 100\% de las trabajadoras sexuales callejeras reporten haber experimentado violencia física, sexual y económica en el ejercicio de su actividad. ${ }^{4}$ Recordemos que la mayoría de los países cuentan con leyes que consideran ilegal algún aspecto del trabajo sexual, estas disposiciones jurídicas se utilizan frecuentemente para justificar el acoso, la extorsión y la violencia contra las trabajadoras sexuales por parte de la policía y de los clientes. ${ }^{1}$

Si bien estas mujeres están expuestas a múltiples manifestaciones de violencia a lo largo de su vida, es de nuestro interés abordar la que ocurre en el contexto del ejercicio de su trabajo sexual y si ésta se asocia con el consumo de alcohol y de otras sustancias, en vista de que también existe un porcentaje muy elevado de uso en esta población. Alrededor del 50\% a 75\% de las trabajadoras sexuales en diferentes contextos y países consumen e incluso abusan del alcohol, ${ }^{6,8,11}$ mientras que en el caso de las drogas, destaca principalmente el uso de cocaína, crack, mariguana y heroína, variando los porcentajes de consumo dependiendo del país y la región. El 78\% de las trabajadoras sexuales en una investigación del Reino Unido ${ }^{9}$ y el 30\% de las participantes en otra realizada en Estados Unidos ${ }^{6}$ reportaron usarlas, al igual que en la zona fronteriza de México, donde 18\% de las mujeres manifestaron haberse inyectado en los últimos 30 días. ${ }^{7}$ El estudio británico señaló que un $32 \%$ usó crack, $17 \%$ cocaína y $61 \%$ mariguana, ${ }^{9}$ mientras que el estadounidense reportó un $80 \%$ de coca y crack y $18.9 \%$ de mariguana. ${ }^{6}$ Las mujeres que trabajaban en la calle reportaron consumos más elevados de droga, lo que habla de condiciones de mayor vulnerabilidad.

La gran cantidad de adversidades y violencias que viven las trabajadoras sexuales llevan a pensar que el uso de las sustancias podría ser un mecanismo para enfrentar el trabajo sexual cotidiano, ${ }^{6}$ aunque también se sabe que el consumo abusivo de sustancias se asocia con experiencias de violencia en la infancia ${ }^{12,13}$ y de violencia de pareja, ${ }^{14}$ en este último caso está particularmente asociada con altos consumos de alcohol. ${ }^{15}$ Este es un trabajo exploratorio que pretende aportar información para realizar estudios más específicos.

\section{MÉTODO}

Se llevó a cabo en 2010 un estudio no experimental y descriptivo con trabajadoras sexuales en el Municipio de Tula de Allende, Estado de Hidalgo, ubicado a 70 kilómetros de la Ciudad de México. 


\section{Participantes}

Un total de 103 trabajadoras sexuales fueron convocadas por un muestreo de conveniencia. Los criterios de selección fueron tener entre 18 y 65 años de edad, realizar trabajo sexual, saber leer y escribir, y aceptar la invitación a participar. De las 103 participantes, el 63.1\% tenía entre 18 y 30 años; en cuanto a la escolaridad, el 30.4\% tenía la primaria o menos, el 39.2\% alcanzó estudios de secundaria, el 21.6\% estudios de bachillerato y el $4.9 \%$ licenciatura no terminada. Respecto a su estado civil, el $49 \%$ eran solteras, el $28 \%$ vivía con una pareja y el $14 \%$ estaban separadas. El 67.0\% provenían de diferentes entidades del país, el 31.0\% de la Ciudad de México y el 2\% eran extranjeras. En el artículo de González et al. que se incluye en este mismo número de SALUD MENTAL, se presenta un cuadro que describe estas características.

\section{Instrumento}

Se utilizó un instrumento diseñado ex profeso que se aplicó por medio de una entrevista semi-estructurada. Para este trabajo se analizaron los siguientes apartados: a) datos sociodemográficos, b) características del trabajo sexual (espacios, actividades que realizan, tiempo de ejercer, horario, razones para ingresar al trabajo sexual y número de clientes), c) problemas de violencia en el trabajo (tipos y actores involucrados) y d) uso de alcohol y drogas.

\section{Procedimiento}

El trabajo de campo se llevó a cabo durante el año 2010 en una Jurisdicción Sanitaria donde las mujeres acudían a revisión médica, dado que es indispensable que se realicen esta revisión para poder obtener el permiso para trabajar. Las entrevistas se realizaron en dicho centro y duraron en promedio de dos a tres horas, previo consentimiento de las mujeres, tras proporcionarles información detallada sobre los objetivos y características de la investigación, y asegurarles la confidencialidad y el anonimato de su participación. Las mujeres podían abandonar el estudio en el momento que así lo desearan y se les aseguró que no tendrían ninguna repercusión en la revisión que realiza la Jurisdicción. El proyecto del que se desprende la información de este artículo fue aprobado por el Comité de Ética del Instituto Nacional de Psiquiatría Ramón de la Fuente Muñiz.

\section{Análisis de la información}

Se realizaron análisis de frecuencias para describir tanto las características sociodemográficas de la muestra como las experiencias de violencia en el entorno laboral y el uso de alcohol y drogas; en el caso de estas últimas se realizó tam- bién un análisis del tipo de sucesos más reportados según lo registrados en respuestas abiertas. Se presentan análisis de $\chi^{2}$ para determinar la asociación entre las experiencias violentas y el consumo de sustancias.

\section{RESULTADOS}

De acuerdo con las entrevistadas, los espacios en los que tiene lugar el trabajo sexual son principalmente bares $(50.8 \%)$, la calle $(39.0 \%)$ y en cabarets $(10.2 \%)$. Las principales actividades que realizan son: a) beber con el cliente, b) bailar con o para el cliente (table dance) y c) realizar trabajo sexual.

Respecto al tiempo de ejercer el trabajo sexual, el 63.0\% tenía entre uno y cinco años y el $24.0 \%$ más de cinco años. El $53.9 \%$ tiene un horario de trabajo mixto (vespertino y nocturno) y para el $46.1 \%$ el horario es nocturno. La jornada de trabajo para más del $40 \%$ es de 40 a 60 horas por semana, mientras que casi el 30\% trabajaba entre 11 y 40 horas.

En cuanto a las razones para ingresar al trabajo sexual el $37.2 \%$ mencionó que lo hizo por comentario o consejo de amigas, el $33.1 \%$ porque no tenía dinero y/o su pareja no tenía trabajo y el $12.3 \%$ porque los encargados de los lugares donde trabajaban como meseras, cajeras, etc., les sugirieron dedicarse al trabajo sexual para mejorar su salario.

El 19.6\% de las mujeres reportó que en la mejor noche tenían en promedio cuatro clientes; seguido del 11.3\% con cinco clientes y el 10.3\% que tenía seis clientes. Mientras que en la peor noche, el $57.3 \%$ mencionó que ninguno y el $28.1 \%$ reportó un cliente.

\section{Experiencias de violencia en el lugar de trabajo}

El 52.4\% de las trabajadoras entrevistadas reportó haber tenido experiencias violentas en el lugar de trabajo. El 95.8\% sufrió algún tipo de violencia física como bofetadas, golpes en el cuerpo, empujones, patadas, jalones de cabello, mordidas, heridas -con armas o con algún otro objeto-; les arrojaron hielos o bebidas, las quemaron con cigarrillos y privaron de la libertad.

El 24.0\% reportó haber sufrido violencia emocional, maltrato psicológico, hostilidad y abuso verbal -insultos, gritos, burlas, críticas, amenazas-, humillación, desprecio, devaluación y represalias por parte de los clientes. Asimismo, presenciaron peleas entre clientes o entre compañeras.

En relación con la violencia sexual, el 26.0\% reportó acoso y abuso sexual, ser forzadas a tener sexo sin protección o en formas no convenidas y violación.

Respecto a los actores involucrados en los episodios violentos, el $97.9 \%$ reportó que fueron las compañeras de trabajo por quitarse los clientes; el 77.2\%, los clientes; el $30.2 \%$, los propietarios o empleados de los bares y el $25.0 \%$ la policía (cuadro 1). 
Cuadro 1. Experiencias violentas en el trabajo sexual ( $N=103)$

\begin{tabular}{|c|c|c|c|}
\hline Actor & Violencia física & Violencia emocional & Violencia sexual \\
\hline $\begin{array}{l}\text { Clientes } \\
77.2 \%\end{array}$ & $\begin{array}{l}\text { - bofetadas } \\
\text { - agresiones } \\
\text { - golpes en cuerpo } \\
\text { - empujones, patadas } \\
\text { - jalones de cabello } \\
\text { - mordidas } \\
\text { - heridas (armas y objetos) } \\
\text { - arrojar hielos o bebidas } \\
\text { - quemaduras con cigarros } \\
\text { - privación de libertad }\end{array}$ & $\begin{array}{l}\text { - maltrato psicológico } \\
\text { - hostilidad y abuso verbal } \\
\text { - insultos, gritos, burlas, críticas, } \\
\text { - amenazas } \\
\text { - humillación, desprecio, deva- } \\
\text { luación } \\
\text { - presenciar peleas entre clientes }\end{array}$ & $\begin{array}{l}\text { - abuso sexual } \\
\text { - acoso sexual } \\
\text { - forzar a tener sexo sin protec- } \\
\text { ción } \\
\text { - forzar a tener sexo en formas } \\
\text { no convenidas } \\
\text { - crítica de funcionamiento sexual } \\
\text { - violación sexual }\end{array}$ \\
\hline $\begin{array}{l}\text { Compañeras } \\
97.2 \%\end{array}$ & $\begin{array}{l}\text { - peleas por los clientes } \\
\text { - golpes en el baño }\end{array}$ & $\begin{array}{l}\text { - represalias } \\
\text { - presenciar peleas entre com- } \\
\text { pañeras }\end{array}$ & \\
\hline $\begin{array}{l}\text { Encargado bar } \\
30.2 \%\end{array}$ & & $\begin{array}{l}\text { - amenazas } \\
\text { - represalias }\end{array}$ & $\begin{array}{l}\text { - abuso } \\
\text { - acoso sexual } \\
\text { - explotación sexual }\end{array}$ \\
\hline $\begin{array}{l}\text { Policías } \\
25.5 \%\end{array}$ & & $\begin{array}{l}\text { - extorsión } \\
\text { - falta de protección }\end{array}$ & $\begin{array}{l}\text { - abuso de autoridad } \\
\text { - manoseo } \\
\text { - acoso sexual }\end{array}$ \\
\hline Pareja/Proxeneta & & - maltrato de pareja & \\
\hline
\end{tabular}

\section{Uso de sustancias}

En relación con el uso de sustancias, el 98\% de las trabajadoras sexuales reportaron haber consumido alcohol en el último mes; el 23.3\%, cocaína y el 11.7\%, mariguana. Cabe señalar que $40 \%$ fuma tabaco.

El 75\% de las trabajadoras sexuales se reporta como bebedora alta, $13 \%$ tiene un consumo frecuente, $9 \%$ tiene un consumo leve, y solamente 3\% no bebe. El 37.6\% consume más de 20 copas por ocasión y el $42.5 \%$ bebe de 12 a 20 copas por ocasión. El 23.5\% bebe diariamente, el 26.5\% casi diario y el $33.3 \%$ bebe de tres a cuatro días a la semana.

El $47.8 \%$ de las mujeres señalaron consumir alcohol para ser sociables, $42 \%$ para enfrentar las tensiones; $39 \%$ mencionaron que beber es parte del negocio porque necesitan ganar más; 37\% lo hicieron para sentir confianza con los hombres, $30.1 \%$ para llenar una necesidad emocional y $16.8 \%$ para tolerar a los clientes borrachos.

El $97 \%$ de las mujeres informaron que generalmente los clientes las presionan para beber alcohol y un porcentaje similar refirió que consumen por las exigencias de los encargados de los lugares para que los clientes consuman más y de esta manera puedan ganar más dinero.

\section{Experiencias de violencia y consumo de sustancias}

Se analizó el reporte de experiencias violentas alguna vez en la vida en general y en el último mes, y de violencia física, sexual y emocional alguna vez en la vida, en relación con el consumo de mariguana, cocaína y tabaco, y el patrón de consumo de alcohol por medio de pruebas de $\chi^{2}$. Dado que el consumo leve tuvo un porcentaje muy bajo, se recodificó junto al frecuente $(21 \%)$ y se comparó con el consumo alto (75\%).

En el cuadro 2 se observa que el haber experimentado alguna vez violencia en el ámbito del trabajo sexual se asoció con un consumo mayor de cocaína (34.6\% vs. el $12.8 \%$ de quienes no la experimentaron) $\left[\chi^{2}=6.417\right.$, $\left.(100,1) \mathrm{gl} \mathrm{p}<.05\right]$ y de tabaco $(54.7 \%$ vs. $27.7 \%)\left[\chi^{2}=7.486(100,1) g l p<.01\right]$. Las mujeres que habían experimentado alguna violencia física en su trabajo reportaron un consumo de alcohol significativamente más alto $(88.6 \%)$ que quienes no la reportaron $(69.6 \%)\left[\chi^{2}=5.180(100,1) g l \mathrm{p}<.05\right]$; aquellas que habían experimentado violencia emocional en este ámbito reportaron más consumo de alcohol de frecuente a leve (50\%) que las mujeres que no la reportaron $(18.2 \%)\left[\chi^{2}=4.514(100,1) \mathrm{gl}\right.$ $\mathrm{p}<.05]$. Las mujeres que experimentaron violencia en el último mes, reportaron significativamente más consumo de cocaína $(40 \%$ vs. $13.6 \%)\left[\chi^{2}=9.074(100,1) g l p<.01\right]$, de tabaco (61\% vs. $28.8 \%)\left[\chi^{2}=10.272(100,1) g l p<.001\right]$ y de consumo alto de alcohol $(90.2 \%$ vs. $69.5 \%)\left[\chi^{2}=6.071(100,1) \mathrm{gl} \mathrm{p}<.05\right]$.

\section{DISCUSIÓN}

Este es un estudio exploratorio que muestra apenas algunos resultados como punto de partida para profundizar en el vínculo entre la violencia que existe en el ámbito del ejercicio del trabajo sexual y el consumo de alcohol, tabaco y drogas ilegales. Sin embargo, consideramos que tiene gran importancia dada la inexistente bibliográfica en nuestro país que 
Cuadro 2. Experiencias violentas en el trabajo y consumo de sustancias ( $N=103$ )

\begin{tabular}{|c|c|c|c|c|c|c|c|c|c|c|c|c|c|c|c|c|c|c|c|c|}
\hline & \multicolumn{4}{|c|}{ Alguna vez } & \multicolumn{4}{|c|}{$\begin{array}{l}\text { Violencia física } \\
\text { alguna vez }\end{array}$} & \multicolumn{4}{|c|}{$\begin{array}{l}\text { Violencia sexual } \\
\text { alguna vez }\end{array}$} & \multicolumn{4}{|c|}{$\begin{array}{c}\text { Violencia emocional } \\
\text { alguna vez }\end{array}$} & \multicolumn{4}{|c|}{ En el último mes } \\
\hline & \multicolumn{2}{|c|}{$\begin{array}{c}\text { No } \\
(n=48)\end{array}$} & \multicolumn{2}{|c|}{$\begin{array}{c}\text { Sí } \\
(\mathrm{n}=52)\end{array}$} & \multicolumn{2}{|c|}{$\begin{array}{c}\text { No } \\
(n=55)\end{array}$} & \multicolumn{2}{|c|}{$\begin{array}{c}\text { Sí } \\
(\mathrm{n}=45)\end{array}$} & \multicolumn{2}{|c|}{$\begin{array}{c}\text { No } \\
(n=86)\end{array}$} & \multicolumn{2}{|c|}{$\begin{array}{c}\text { Sí } \\
(n=14)\end{array}$} & \multicolumn{2}{|c|}{$\begin{array}{c}\text { No } \\
(n=88)\end{array}$} & \multicolumn{2}{|c|}{$\begin{array}{c}\text { Sí } \\
(n=12)\end{array}$} & \multicolumn{2}{|c|}{$\begin{array}{c}\text { No } \\
(n=59)\end{array}$} & \multicolumn{2}{|c|}{$\begin{array}{c}\text { Sí } \\
(\mathrm{n}=41)\end{array}$} \\
\hline & $f$ & $\%$ & $f$ & $\%$ & $f$ & $\%$ & $f$ & $\%$ & $f$ & $\%$ & $f$ & $\%$ & $f$ & $\%$ & $f$ & $\%$ & $f$ & $\%$ & $f$ & $\%$ \\
\hline Consumo de mariguana & 3 & 6.4 & 9 & 17.0 & 4 & 7.3 & 8 & 17.8 & 9 & 10.5 & 3 & 21.4 & 10 & 11.4 & 2 & 16.7 & 4 & 6.8 & 8 & 19.5 \\
\hline Consumo de cocaína & 6 & 12.8 & 18 & $34.6 *$ & 11 & 20 & 13 & 29.5 & 19 & 22.4 & 5 & 35.7 & 21 & 23.9 & 3 & 27.3 & 8 & 13.6 & 16 & $40 * *$ \\
\hline Consumo de tabaco & 13 & 27.7 & 29 & $54.7 * *$ & 19 & 34.5 & 23 & 51.1 & 36 & 41.9 & 6 & 42.9 & 34 & 38.6 & 8 & 66.7 & 17 & 28.8 & 25 & $61 * * *$ \\
\hline $\begin{array}{l}\text { Patrón de consumo } \\
\text { de alcohol }\end{array}$ & & & & & & & & & & & & & & & & & & & & \\
\hline - Alto & 34 & 70.8 & 44 & 84.6 & 39 & 69.6 & 39 & $88.6 *$ & 66 & 76.7 & 12 & 85.7 & 72 & 81.8 & 6 & 50 & 41 & 69.5 & 37 & 90.2 \\
\hline - Frecuente a leve & 14 & 29.2 & 8 & 15.4 & 17 & 30.4 & 5 & 11.4 & 20 & 23.3 & 2 & 14.3 & 16 & 18.2 & 6 & $50 *$ & 18 & 30.5 & 4 & $9.8 *$ \\
\hline
\end{tabular}

${ }^{* * *} p<.001 .{ }^{* *} p<.01 .{ }^{*} p<.05$.

aborde a estas mujeres en cuanto a sus condiciones de vida y los problemas de salud física y mental que experimentan. Es una manera de visibilizar a esta población estigmatizada y dar cuenta de sus múltiples necesidades.

Como se observó, la mitad de las trabajadoras sexuales entrevistadas tiene como centro de su actividad los bares, y dos quintas partes trabaja en la calle en horarios vespertino y nocturno por muchas horas y llevan ya varios años dedicadas al sexoservicio. Destaca que las razones para involucrarse en el trabajo sexual sean principalmente económicas, habiendo sido aconsejadas por amigas o personas donde laboraban en que se involucraran en esta actividad. A diferencia de lo que ocurre en otras latitudes, no se reporta ningún caso que se dedique al sexo servicio con el fin de comprar drogas. ${ }^{6,9}$ De este modo, es la necesidad económica el principal motivo de estas trabajadoras sexuales.

Destaca que, como se reporta en otros estudios, efectivamente estas mujeres laboran en condiciones de violencia que las hacen extremadamente vulnerables. La mitad de las trabajadoras sexuales han estado expuestas a violencia, particularmente de tipo físico, pero también la de tipo sexual, lo que concuerda con otros estudios, aunque son victimizadas de múltiples maneras. Si bien reportan la violencia por parte de los clientes, destaca que señalen a sus compañeras trabajadoras sexuales como las principales fuentes de violencia, a lo que se agregan también los dueños de bares y la policía.

En relación con el uso de sustancias, prácticamente todas las trabajadoras sexuales entrevistadas reportaron haber consumido alcohol en el último mes, un porcentaje extremadamente elevado considerando además la prevalencia reportada por mujeres en población general. Este consumo parece verse promovido por su alta disponibilidad y la fuerte presión que ejercen tanto clientes como dueños de bares para hacerlo mientras trabajan, sin embargo habrá que analizar en otro momento el papel que juegan otras violencias que ocurren con su pareja y a lo largo de la historia.

En cuanto al consumo de otras drogas, este trabajo concuerda con que las más utilizadas son la cocaína y la mariguana, no existiendo en esta población el consumo de heroína como ocurre en otros países o en las zonas fronterizas. ${ }^{7}$ Sin embargo, destacó como un problema importante el tabaco, el que también podría fungir como un mecanismo de enfrentamiento ante la ansiedad.

Las limitaciones de este estudio son el tamaño de la muestra, su selección intencional y el instrumento, de manera que es posible que los resultados no puedan generalizarse a todas las trabajadoras sexuales de la entidad y que algunos de ellos presenten algunos problemas de validez por haber sido recolectados con un instrumento elaborado ex profeso que no se había probado previamente.

Aun así, este trabajo aporta información valiosa para ser considerada por los servicios de salud a los que acuden estas mujeres necesariamente para poder seguir trabajando. Se requeriría explorar y atender las múltiples violencias que viven y ofrecer tratamiento para el abuso y dependencia al alcohol, tabaco, cocaína y mariguana, de manera respetuosa y sin violentar sus derechos humanos. Si bien destaca la importancia de abordar el contexto en el que viven de manera estructural, al menos podría plantearse un acceso a la atención en salud que posibilite el romper el ciclo de violencia y mejorar en lo posible su salud física y mental atendiéndolas adecuadamente como las ciudadanas que son.

\section{REFERENCIAS}

1. ONUSIDA. Nota de orientación del ONUSIDA sobre el VIH y el trabajo sexual. UNAIDS; Ginebra: 2009; p.27.

2. UNAIDS. Technical update on sex work and HIV/AIDS. Ginebra: 2002.

3. Shannon K, Kerr T, Allinott S, Chettiar J et al. Social and structural violence and power relations in mitigating HIV risk of drug-using women in survival sex work. Social Science Medicine 2008;66(4):911-921. 
4. Sanders T, Campbell R. Designing out vulnerability, building in respect: violence, safety and sex work policy. British J Sociology 2007;58(1):1-19.

5. Goodyear MD, Cusick L. Protection of sex workers. BMJ: British Medical J 2007;334: http//dx.doi.org/10.1136/mnj.39087.642801.BE (publicado el 11 de enero de 2007. Consultado el 21 de mayo de 2014).

6. El-Bassel N, Witte SS, Wada T, Gilbert L. Correlates of partner violence among female street-based sex workers: substance abuse, history of childhood abuse, and HIV risks. AIDS Patient Care STDs 2001;15(1):41-51.

7. Ulibarri MD, Strathdee SA, Lozada R, Magis-Rodriguez C et al. Intimate partner violence among female sex workers in two Mexico-US Border cities: Partner characteristics and HIV risk behaviors as correlates of abuse. Psychological Trauma Theory Research Practice Policy 2010;2(4):318-325.

8. Surratt HL, Inciardi JA, Kurtz SP, Kiley MC. Sex work and drug use in a subculture of violence. Crime Delinquency 2004;50(1):43-59.

9. Church S, Henderson M, Barnard M, Hart G. Violence by clients towards female prostitutes in different work settings: questionnaire survey. BMJ British Medical J 2001: http//dx.doi.org/10.1136/ bmj.322.7785.524 (publicado el 3 de marzo del 2001. Consultado el 23 de mayo del 2014.
10. Decker MR, Pearson E, Illangasekare SL, Clark E. Violence against women in sex work and HIV risk implications differ qualitatively by perpetrator. BMC Public Health 2013;13: htt//dx.doi.org/10.1186/14712458/13/876

11. Mooney A, Kidanu A, Bradley HM, Kumoji EK. Work-related violence and inconsistent condom use with non-paying partners among female sex workers in Adama City, Ethiopia. BMC Public Health 2013;13(1):110.

12. Kendler KS, Bulik CM, Silberg J, Hettema JM. Childhood sexual abuse and adult psychiatric and substance use disorders in women: an epidemiological and cotwin control analysis. Archives General Psychiatry 2000;57(10):953-959.

13. Del Río FP, Guardiola MM. Abuso sexual en la infancia y la drogodependencia en la edad adulta. Papeles Psicólogo 2013;34(2):144-149.

14. Tolman RM, Rosen D. Domestic violence in the lives of women receiving welfare mental health, substance dependence, and economic well-being. Violence Against Women 2001;7(2):141-158.

15. Devries KM, Child JC, Bacchus LJ, Mak J et al. Intimate partner violence victimization and alcohol consumption in women: a systematic review and meta-analysis. Addiction 2014;109(3):379-391.

Artículo sin conflicto de intereses 